\title{
A new estimator of the deceleration parameter from galaxy rotation curves
}

\author{
Maurice H.P.M. van Putten
}

Physics and Astronomy, Sejong University, Seoul

https://doi.org/10.5281/zenodo. 162267

KIAS November 12016

Korean National Research Foundation under Grants 2015R1D1A1A01059793 and 2016R1A5A1013277
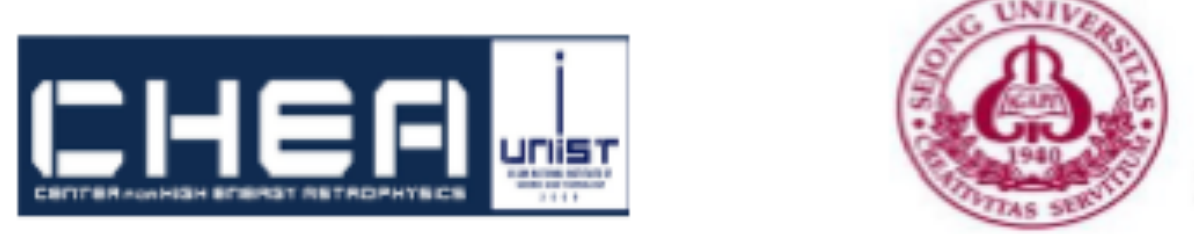

NRFF 한국연구재단 


\section{Outline}

Modern ideas on spacetime

Horizon surfaces and Rindler inertia

The accelerating Universe

Conclusions and outlook 
We are just beginning to probe the nature of spacetime.. 


\section{Attractive gravity on galactic scales}

\section{Repulsive gravity on cosmological scales}

Gravitation governs cosmological evolution from the Big Bang to the present and the distant future. .

$$
\frac{V_{\text {attr }}}{V_{\text {rep }}} \cong 10^{-14}
$$




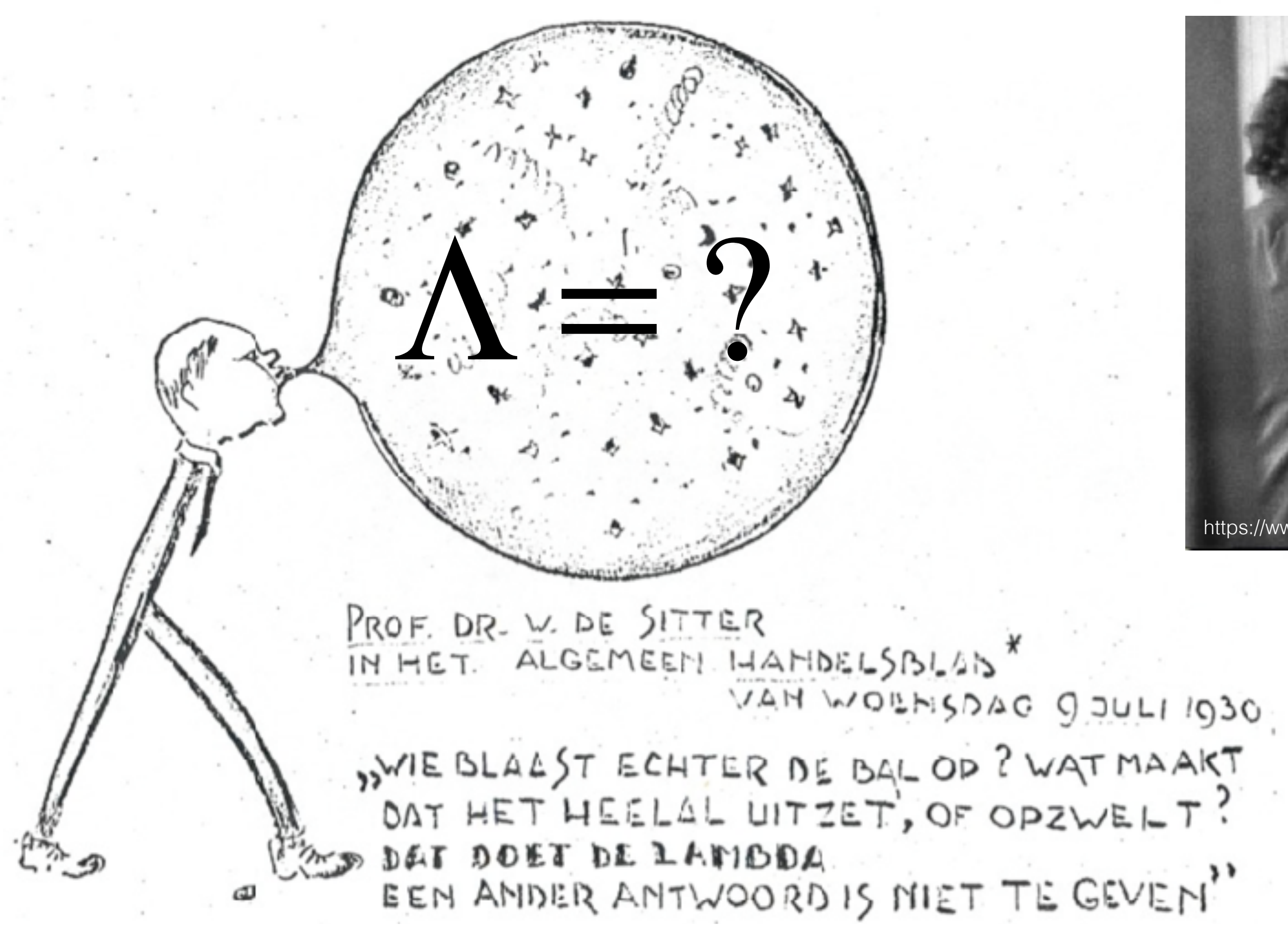

WHO INFLATES THE BALL? WHAT MAKES THE UNIVERSE EXPAND OR SWELL? IHAT'S [BY] LAMBDA, THERE IS NO ALTERNATIVE 


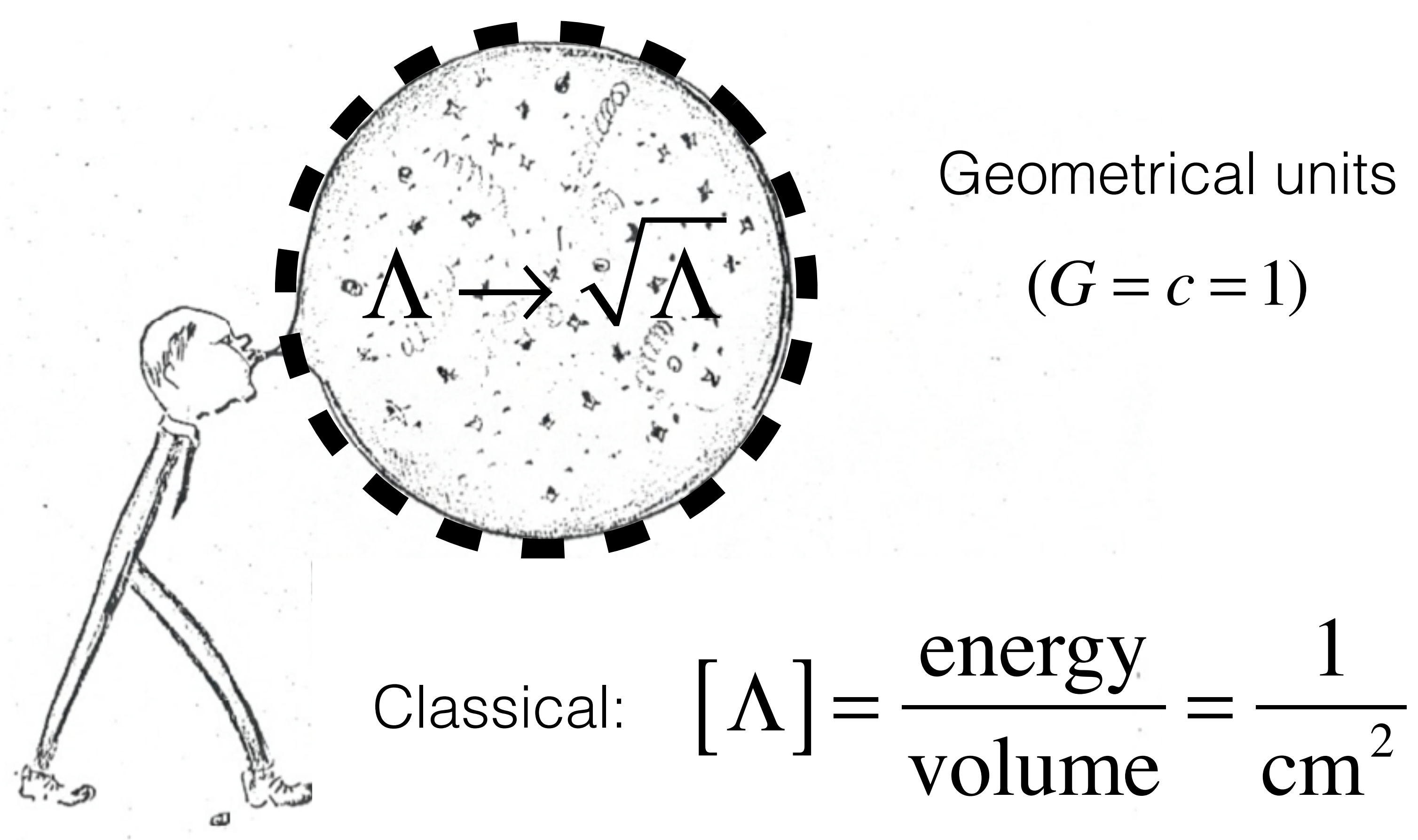

Holographic: $[\sqrt{\Lambda}]=\frac{\text { energy }}{\text { area }}=\frac{1}{\mathrm{~cm}}$ 


\section{Horizons of accelerating observers}

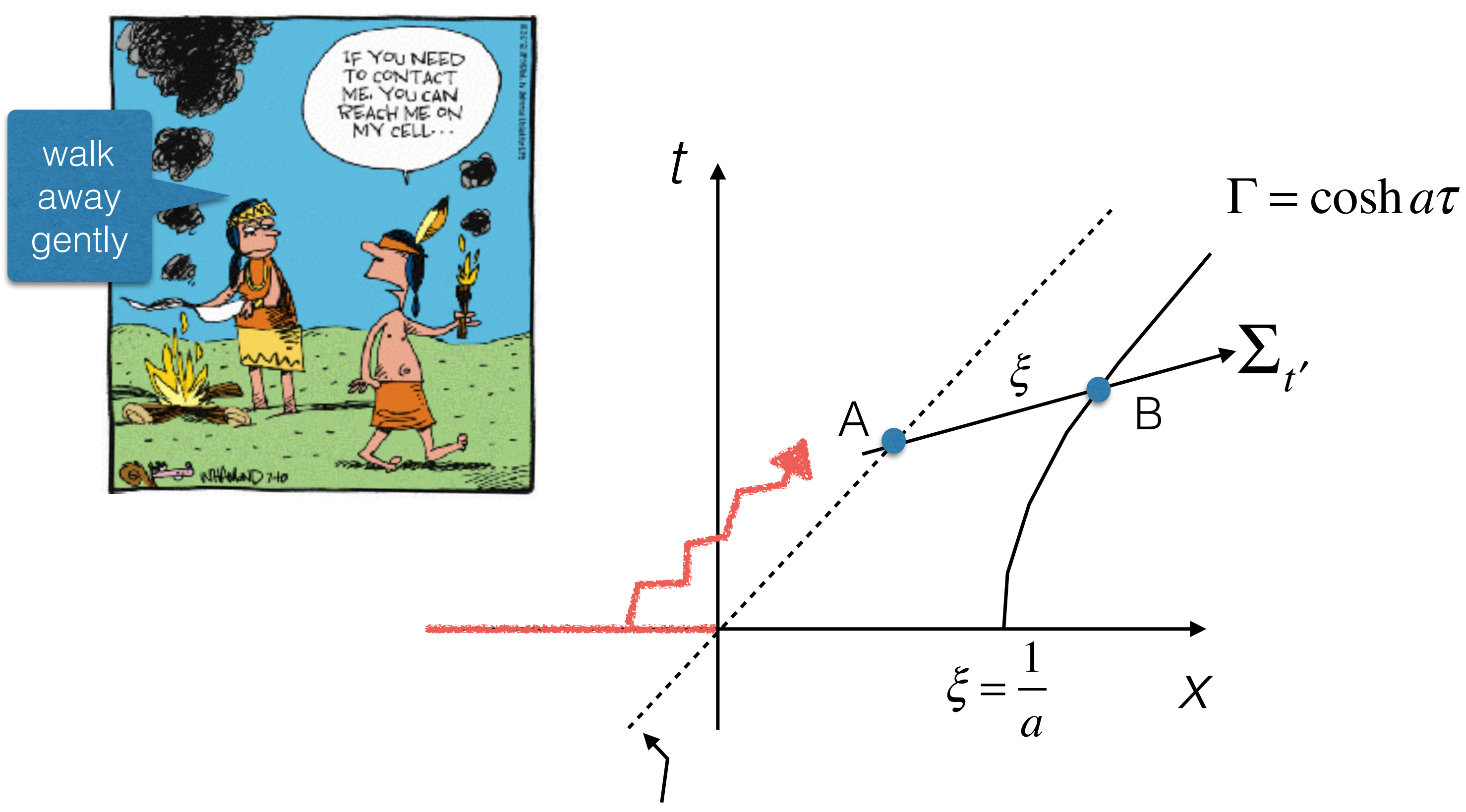

totally "black screen" when looking back 


\title{
Excising a section of Minkowski spacetime
}

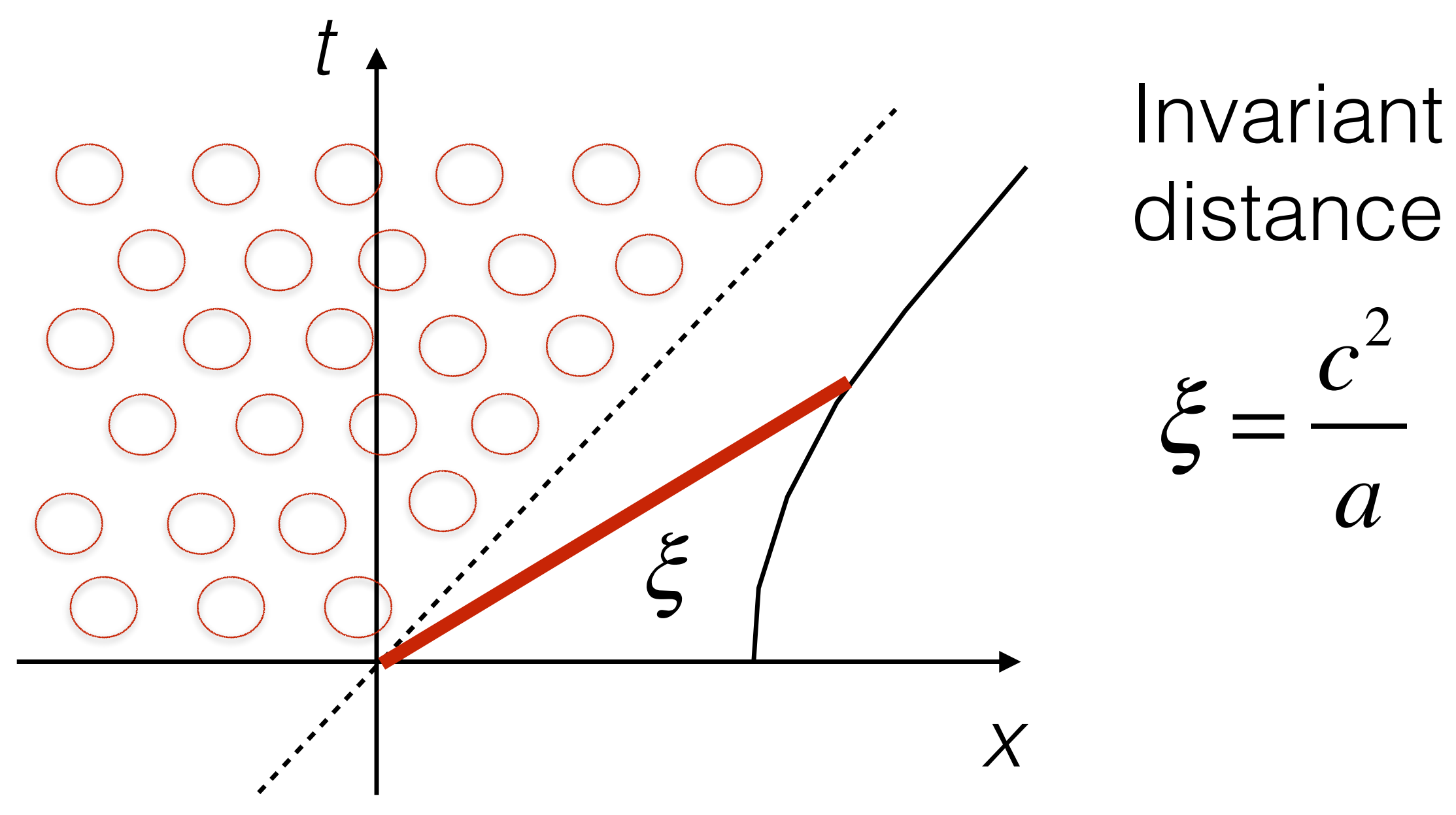

\author{
Light cone in Minkowski spacetime is a \\ Rindler horizon to an accelerating observer
}




\section{Newtonian regime...}

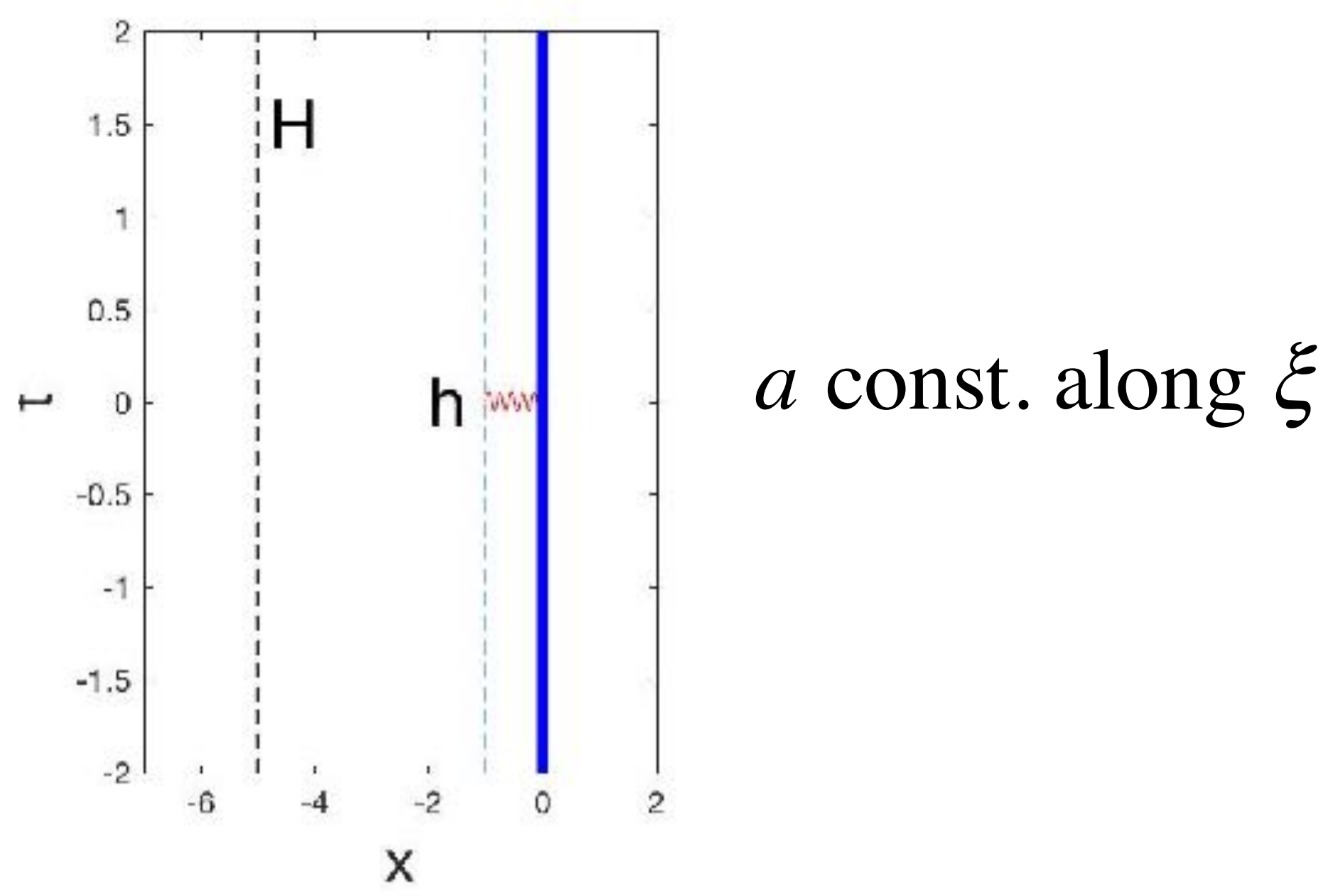




\section{Holographic origin Rindler inertia}

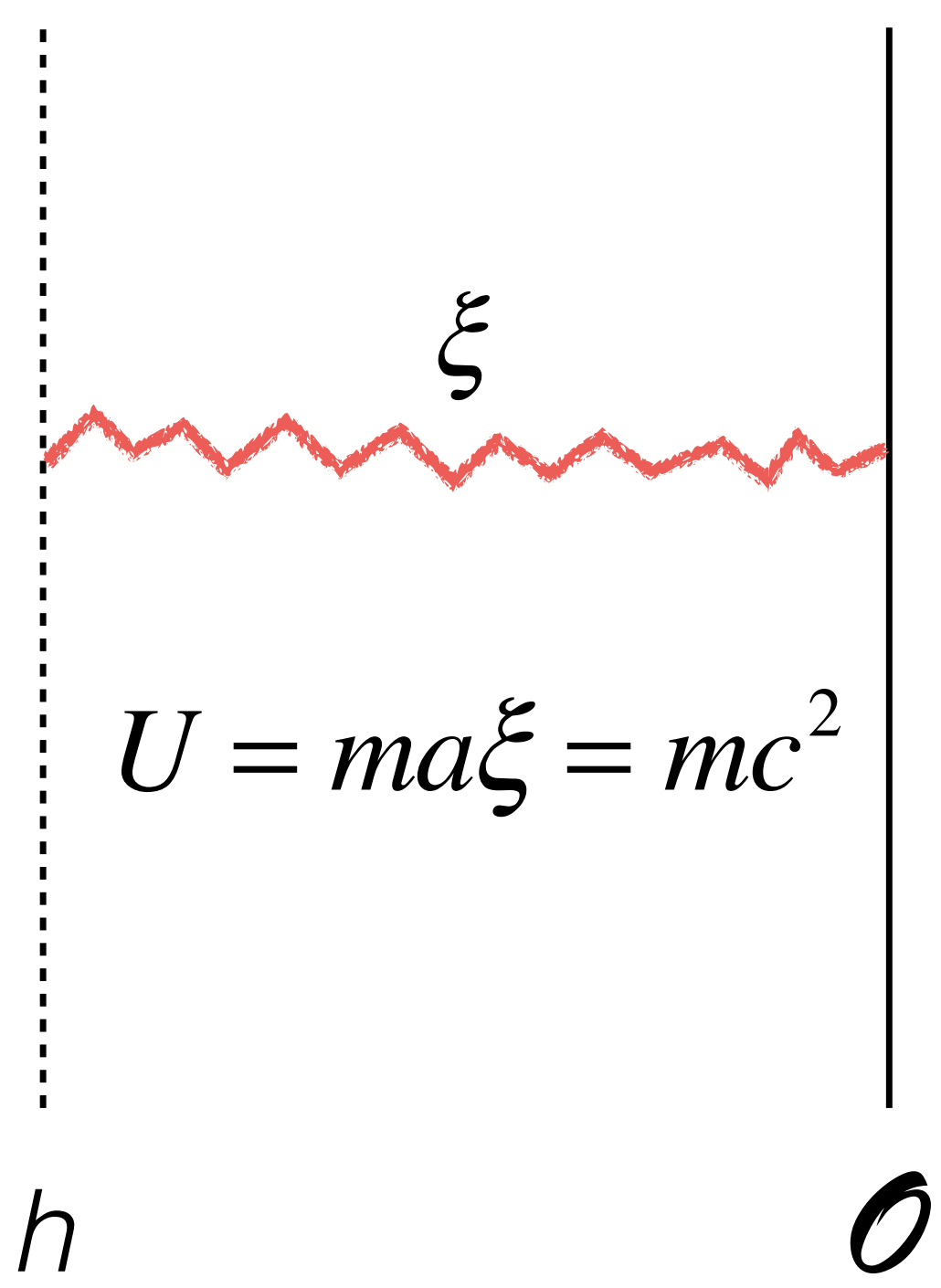

Unitary holography

$$
\begin{aligned}
& I=2 \pi m \xi, \quad d S=-k_{B} d I \\
& k_{B} T=\left(\frac{\partial S}{\partial m c^{2}}\right)^{-1}=\frac{a \hbar}{2 \pi c}=k_{B} T_{U} \\
& F=-T\left(\frac{\partial S}{\partial \xi}\right)=m a
\end{aligned}
$$

O's inertia originates in $h=h(a)$

(van Putten 2012, PRD, 85 064046; 2015 IJMPD 24 1550024; 2016, submitted) 


\section{Collusion Rindler and cosmological horizons}

Observed is $h$ Observed is $H$

$$
\begin{gathered}
\xi=\frac{c^{2}}{a} \\
\sim \\
R_{H}=\frac{c}{H_{0}}
\end{gathered}
$$
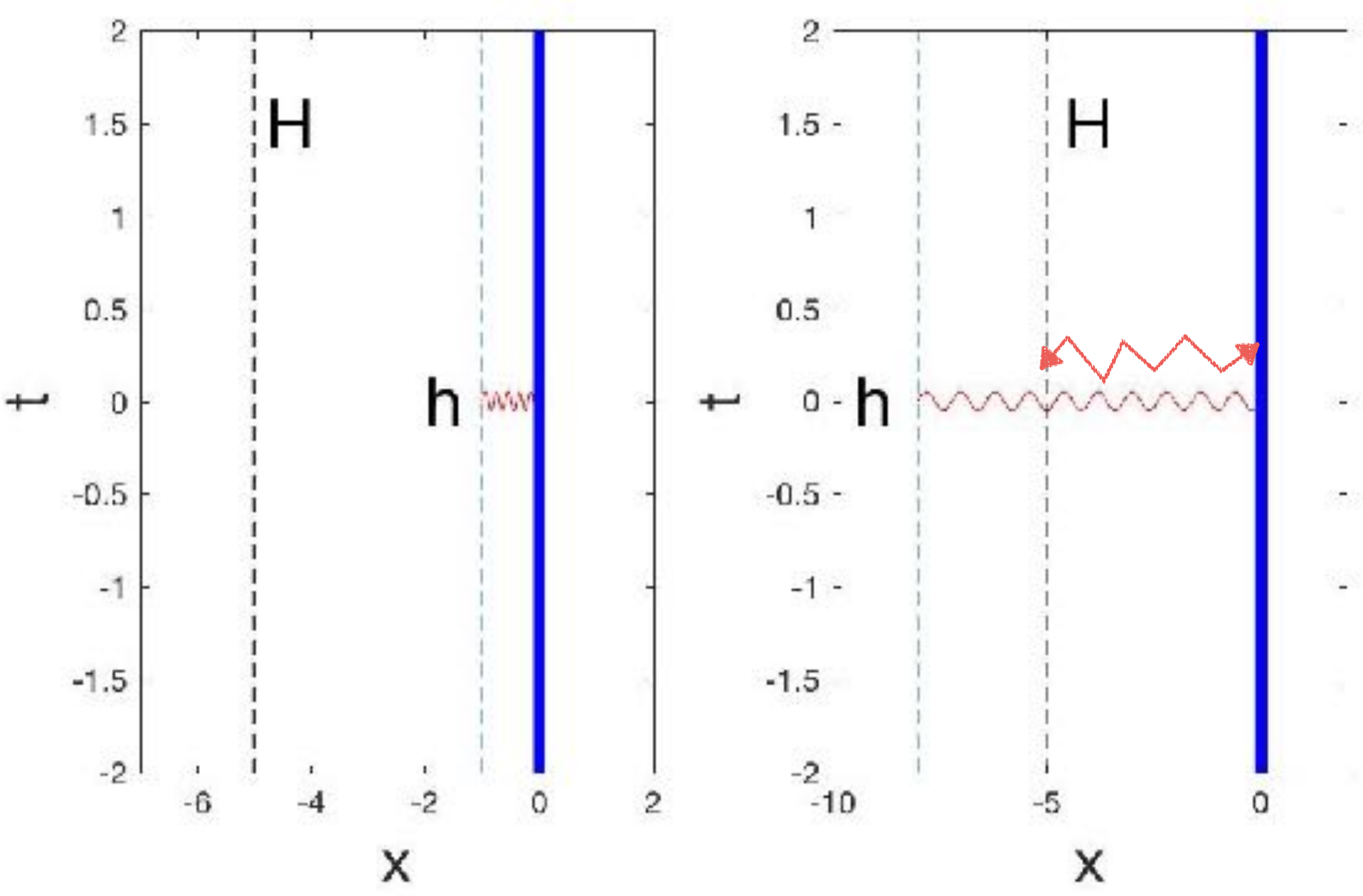

Newtonian inertia Perturbed inertia (reduced) 


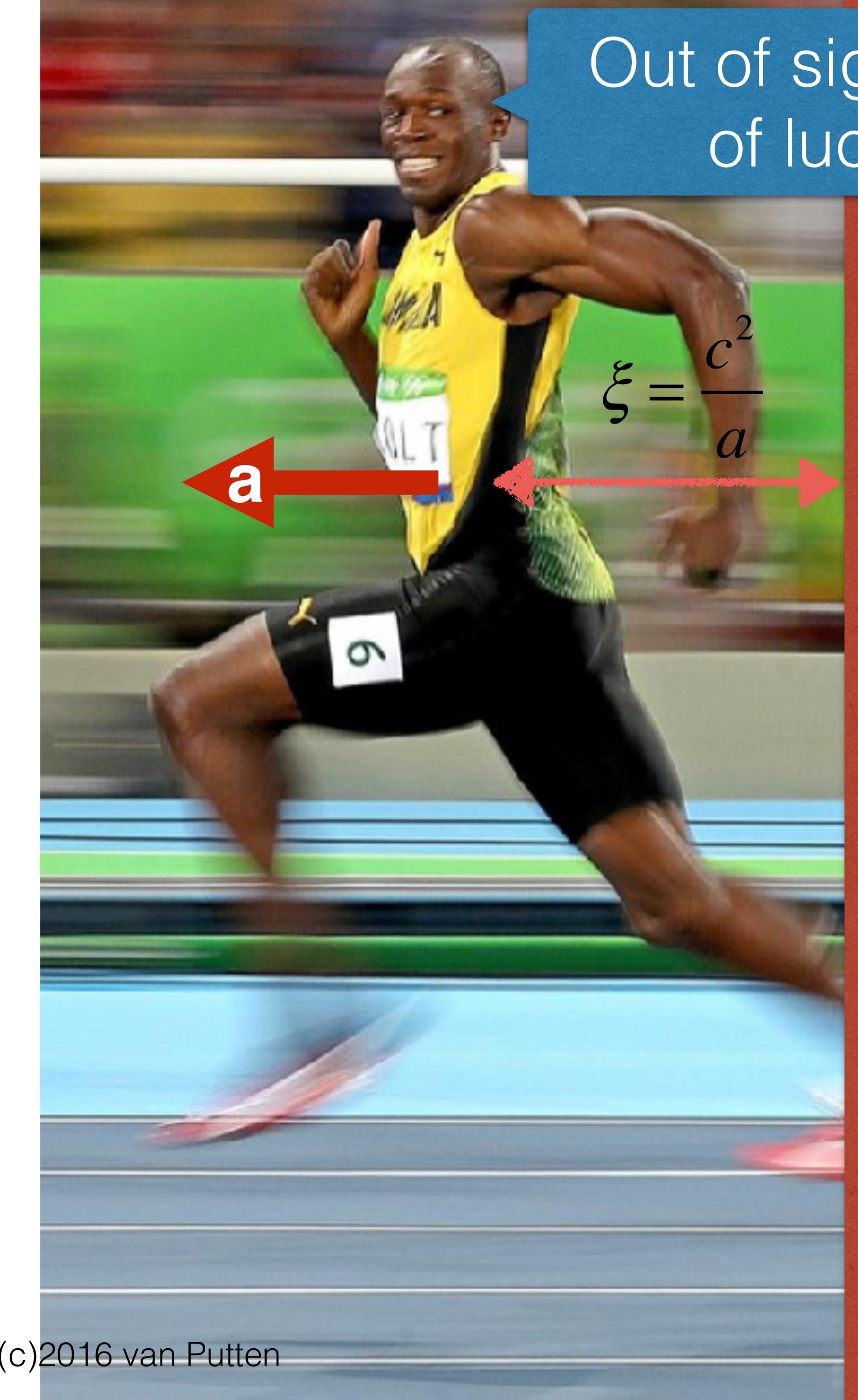

\section{out} k:)

(c)2016 van Putten 


\section{Perturbed Rindler inertia at small accelerations}

Scale of all dark matter and dark energy observations:

$$
a \sim c H_{0} \sim 1 \mathrm{As} \mathrm{s}^{-2}
$$

Relevant to most of the Universe:

$$
\frac{V_{\text {attr }}}{V_{\text {rep }}} \cong 10^{-14}
$$




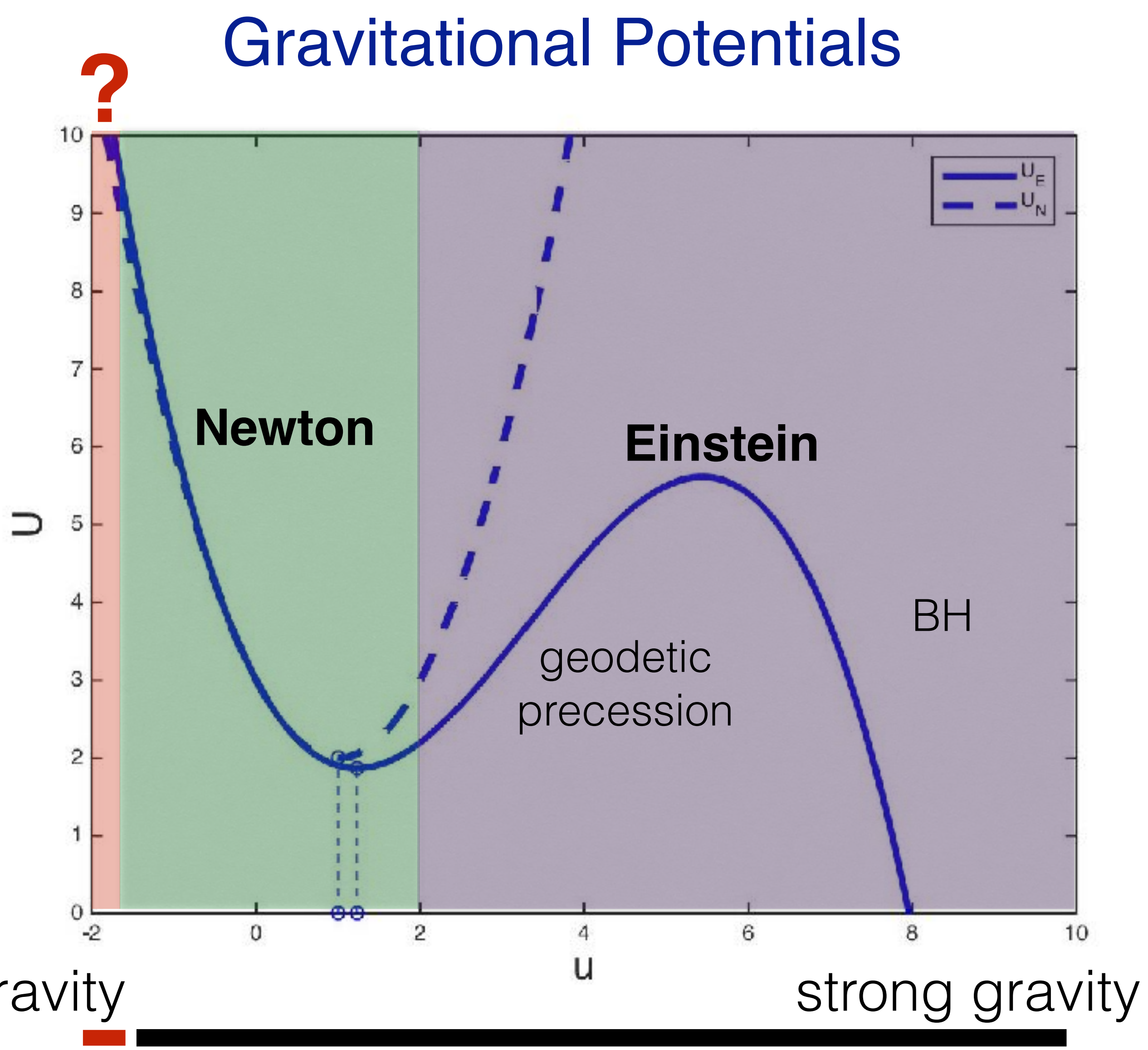




\section{Gravitational Potentials}

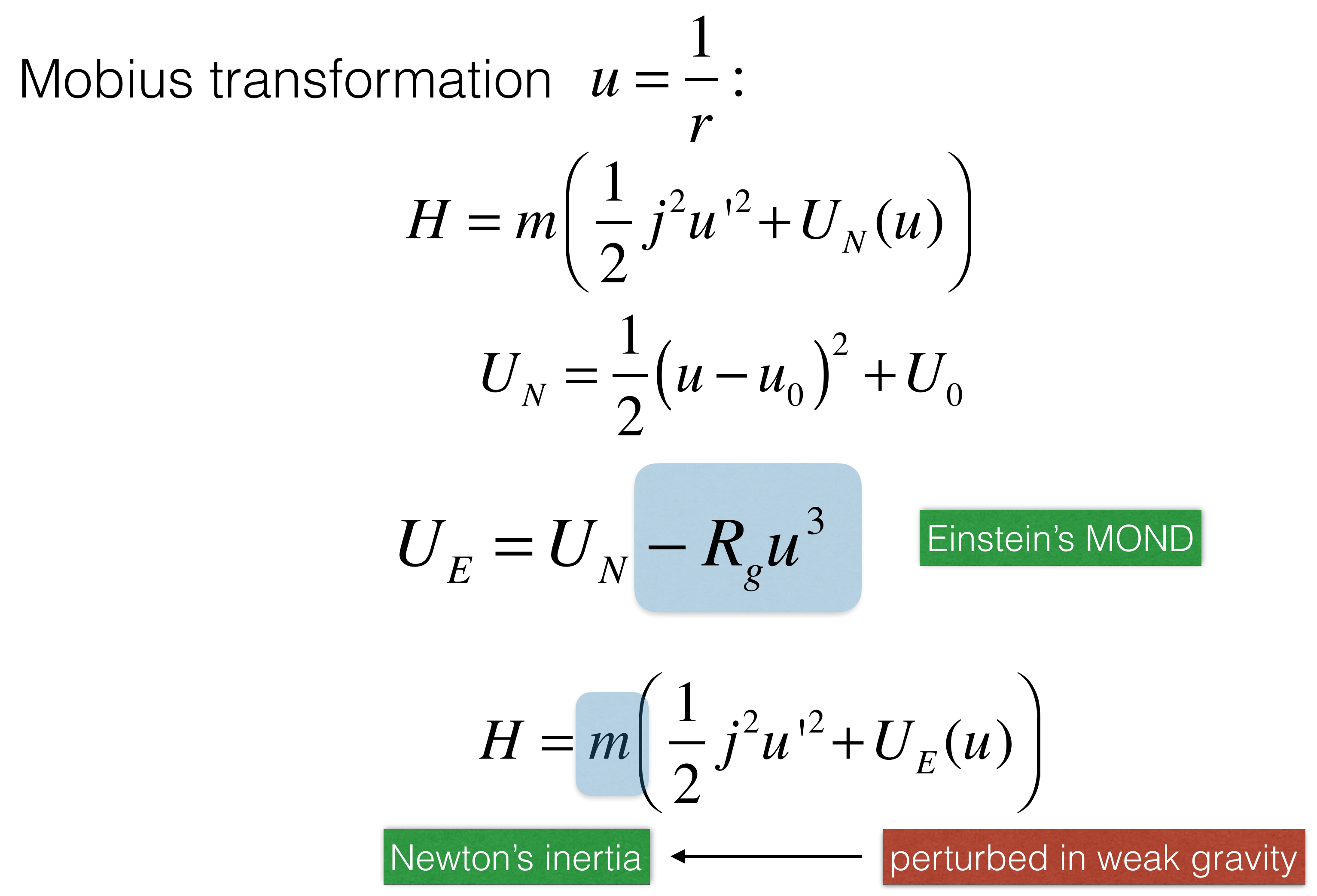




\section{Weak gravity}

\section{Galaxy rotation curves}

Holographic transition radius: $4 \pi r^{2} \sqrt{\Lambda} \cong M$

$$
r_{t}=\sqrt{R_{g} R_{H}}=4.7 \mathrm{kpc} M_{11}^{1 / 2}
$$




\section{Galaxy rotation curves}

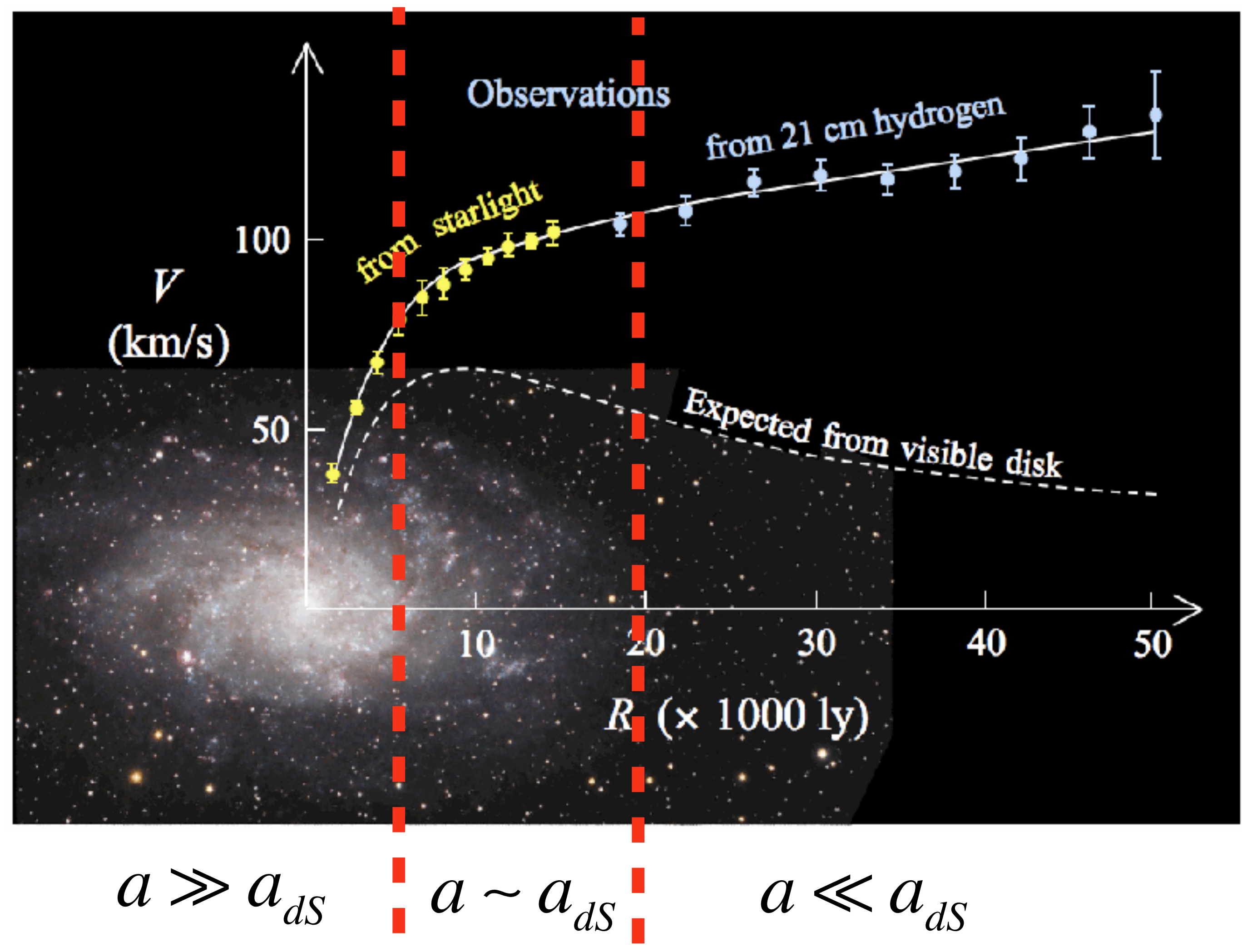




\section{Weak gravity in cosmology}

Dark energy:

$$
\begin{aligned}
& \Omega_{\Lambda}=0.692 \pm 0.010 \\
& H_{0}=67.80 \pm 0.77
\end{aligned}
$$

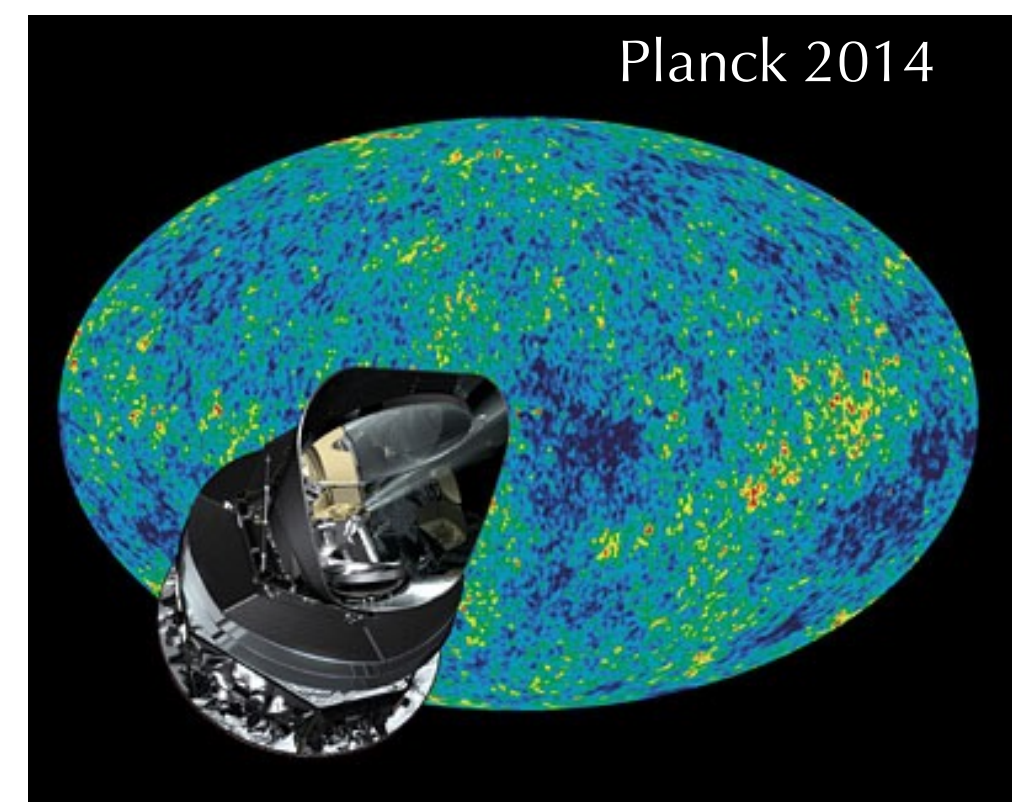

Accelerated expansion (de Sitter when $q=-1$ )

$$
q=\frac{1}{2} \Omega_{M}-\Omega_{\Lambda}<0 \quad(\text { three-flat FRW universe })
$$

(GR on classical vacuum with no hidden low energy scales) 


\section{Intrinsic curvature of cosmological event horizon}

$$
\begin{array}{ll}
a_{H}=\frac{1}{2}(1-q) H \quad \text { Intrinsic surface gravity } \\
\omega_{0}^{2}=(1-q) H^{2} \quad \text { Eigenfrequency on } H
\end{array}
$$

Dynamical dark in four-dimensional spacetime within

$$
\Lambda=(1-q) H^{2}
$$




\section{Weak gravity}

Galaxy rotation curves

$$
r_{t}=\sqrt{R_{g} R_{H}}=4.7 \mathrm{kpc} M_{11}^{1 / 2}
$$

Dynamical dark energy $\quad \Lambda=(1-q) H^{2}$ 


\section{Modified FRW equations}

$$
G_{a b}=8 \pi T_{a b}-(1-q) H^{2} g_{a b}
$$

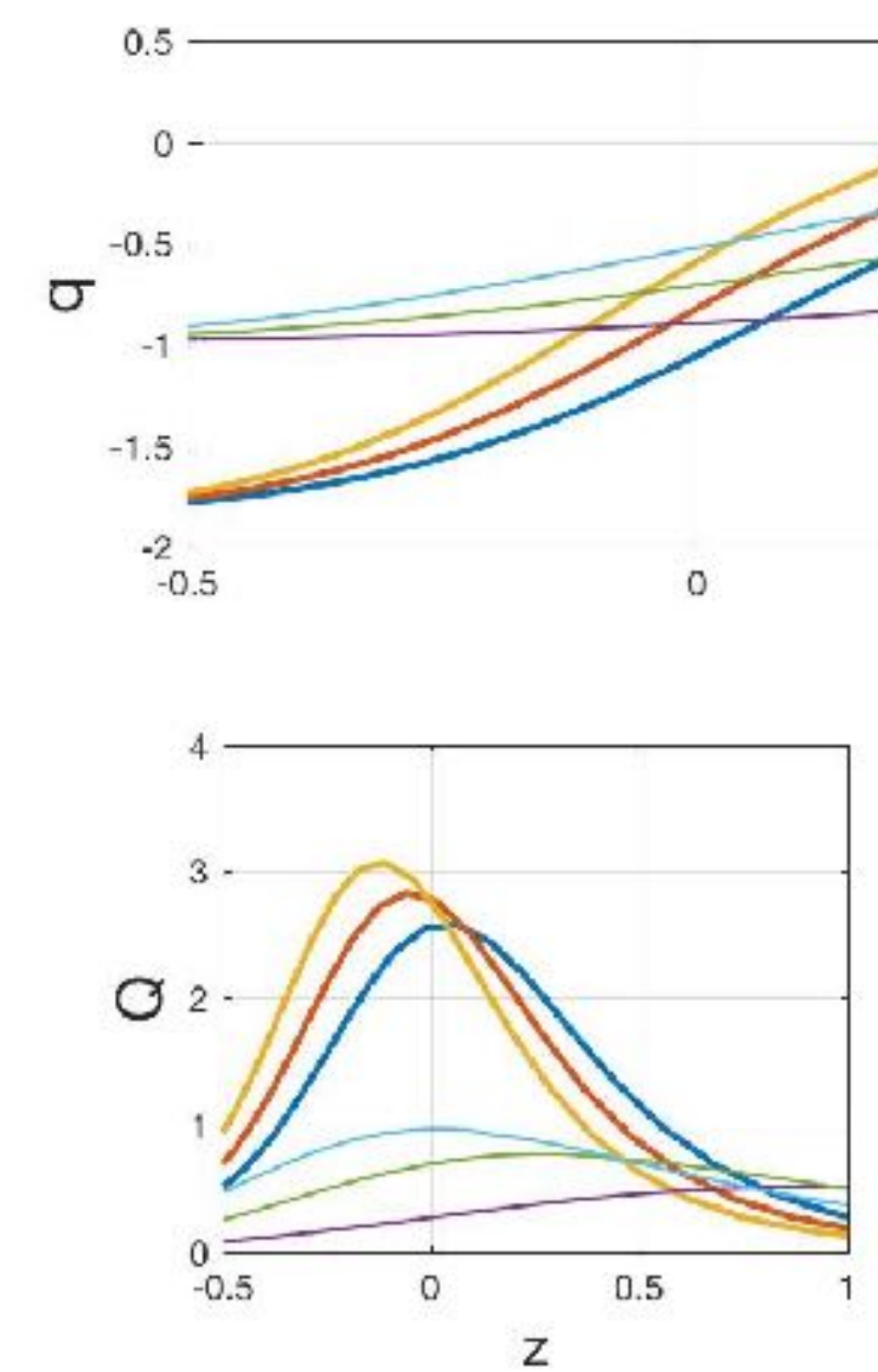

$$
\begin{aligned}
& \Omega_{M}=\frac{1}{3}(2+q) \\
& \Omega_{\Lambda}=\frac{1}{3}(1-q)
\end{aligned}
$$

Z

$$
\begin{aligned}
& Q(z)=\frac{d q(z)}{d z}=2(1-q)\left(H^{-1} \frac{d H}{d z}-a_{0}^{-1}(z) \frac{d a_{0}(z)}{d z}\right) \\
& Q_{0}=\left(\frac{d q(z)}{d z}\right)_{z=0} \quad \text { van Putten } 2015 \text { MNRAS } 450 \text { L48 }
\end{aligned}
$$

$Q_{0}[\mathrm{DDE}]>2.5, \quad Q_{0}[\Lambda \mathrm{CMD}]<1$ 


\section{Tully-Fisher relation}

Observed:

$$
\begin{gathered}
\alpha=\frac{1}{2}, \frac{V_{c}^{2}}{r}=\frac{M_{b}^{\frac{1}{2}}}{r} \quad\left(a_{c} \ll a_{d S}\right) \\
M_{b}=A V_{c}^{4}
\end{gathered}
$$

$A=47 \pm 6 M_{\odot} \mathrm{km}^{-4} \mathrm{~s}^{-4}$

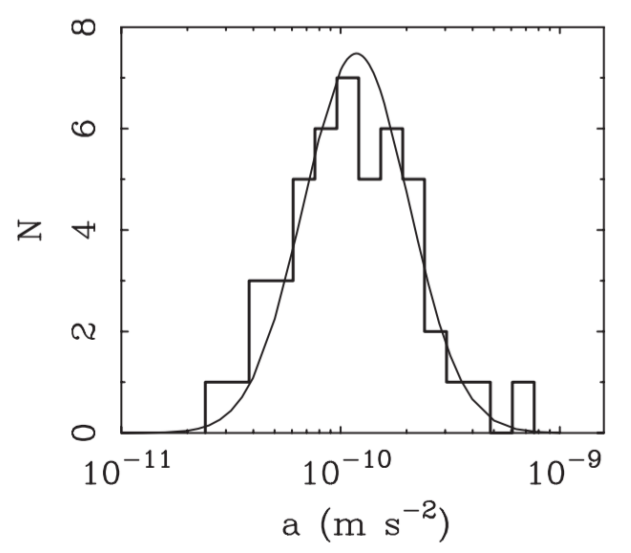

$$
V_{c}^{4}=a_{0} G M_{b}
$$

$$
a_{0} \sim a_{d s}
$$

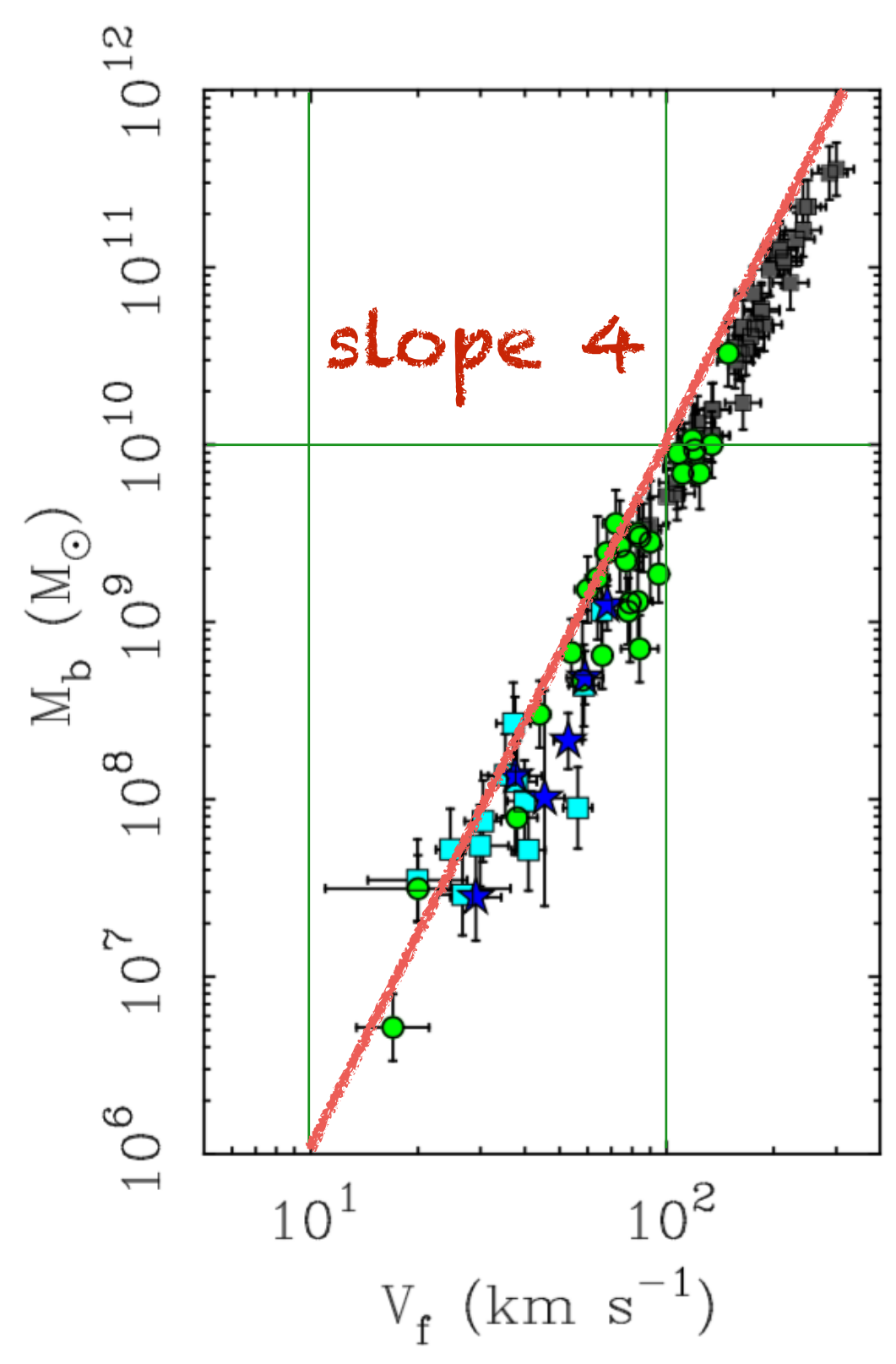

McGaugh, S.S., 2011, Astron. J., 143, 40; PRL, 106, 121303 


\section{Equivalent Milgrom's scale of acceleration ao}

Dimensional reduction by Milgrom's square root:

$$
\begin{gathered}
a=\sqrt{a_{0} a_{N}}, a_{N}=\frac{G M}{r^{2}} \\
a_{0}=\frac{c H}{\sqrt{2} \pi}\left(\frac{1-q}{2}\right)^{\frac{1}{2}} \cong 1.2 h_{0}\left(\frac{1-q}{1.8}\right)^{\frac{1}{2}} \AA^{\circ} \mathrm{s}^{-2}
\end{gathered}
$$




\section{Galaxy rotation curves}

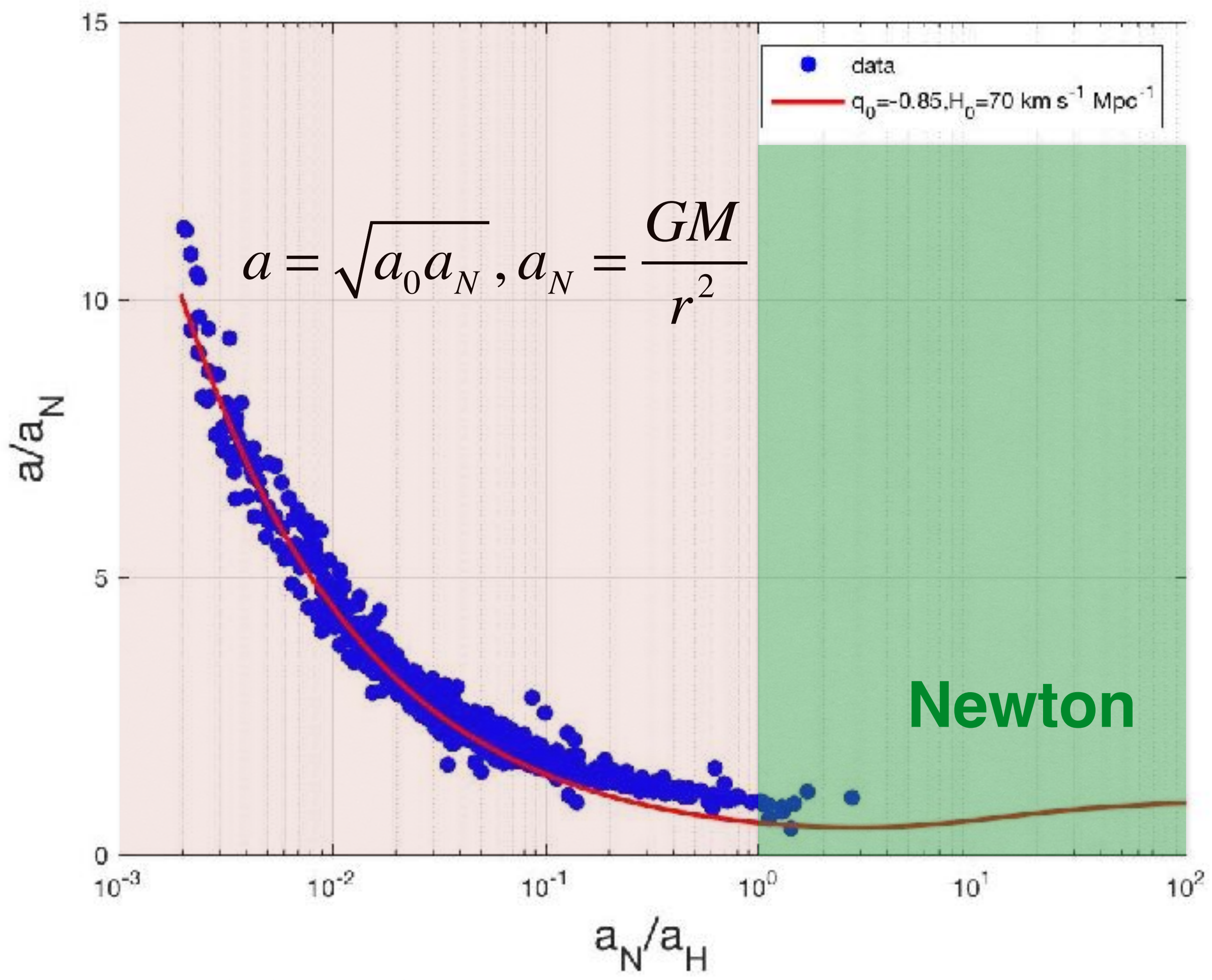

Data from S.S. McGaugh hitp://astroweb.case.edu/ssm/datalaMDaccRgn-LR.dat, rescaled to $\mathrm{H} 0=70 \mathrm{~km} \mathrm{~s} \mathrm{Mpc}$ 


\section{Weak gravity}

Galaxy rotation curves

$$
r_{t}=\sqrt{R_{g} R_{H}}=4.7 \mathrm{kpc} M_{11}^{1 / 2}
$$

Dynamical dark energy

$$
\Lambda=(1-q) H^{2}
$$

Deceleration parameter

$$
q=1-\left(\frac{4 \pi a_{0}}{c H}\right)^{\frac{1}{2}}
$$




\section{Deceleration parameter $q_{0} \sim-0.85$}

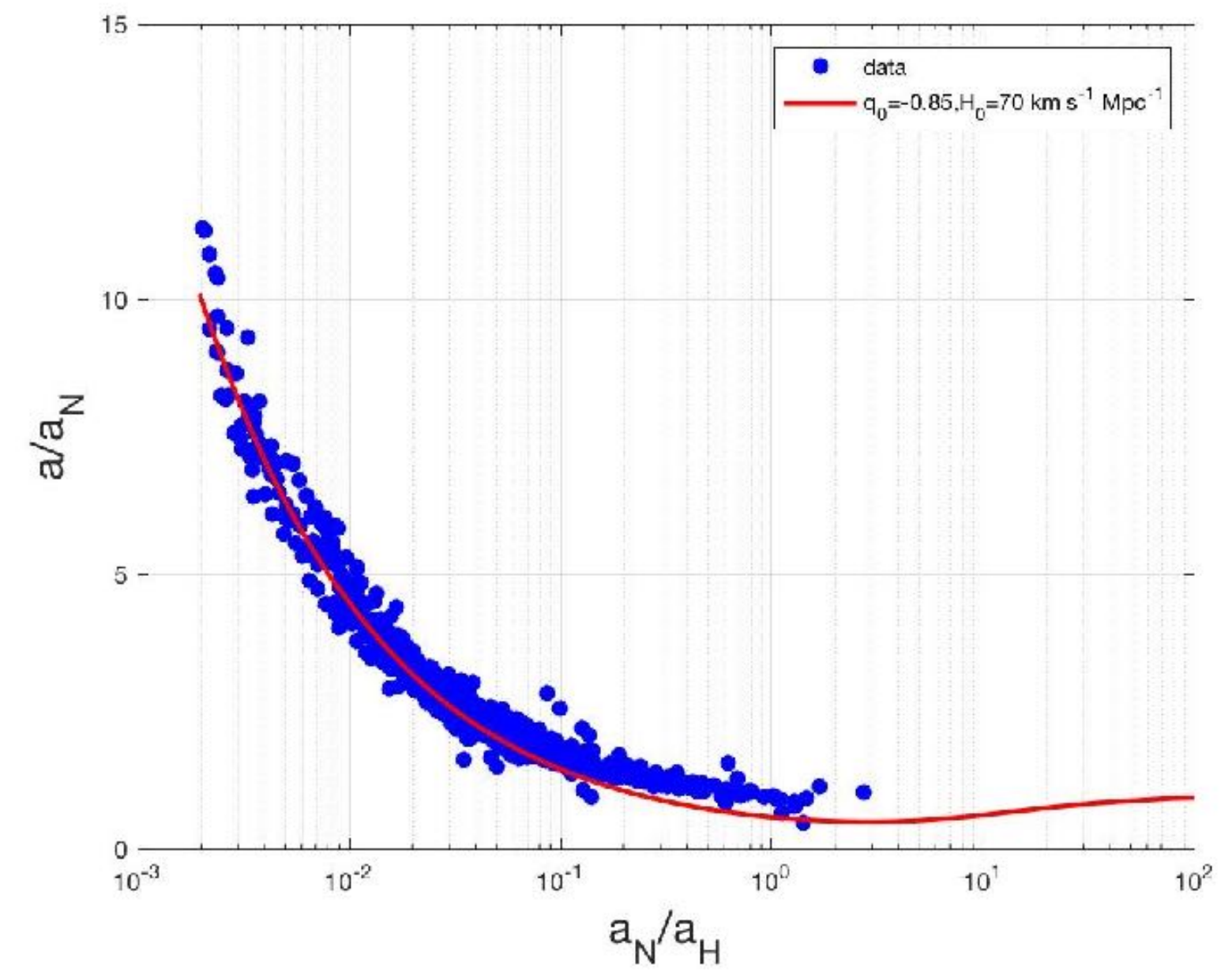

- theoretical curve

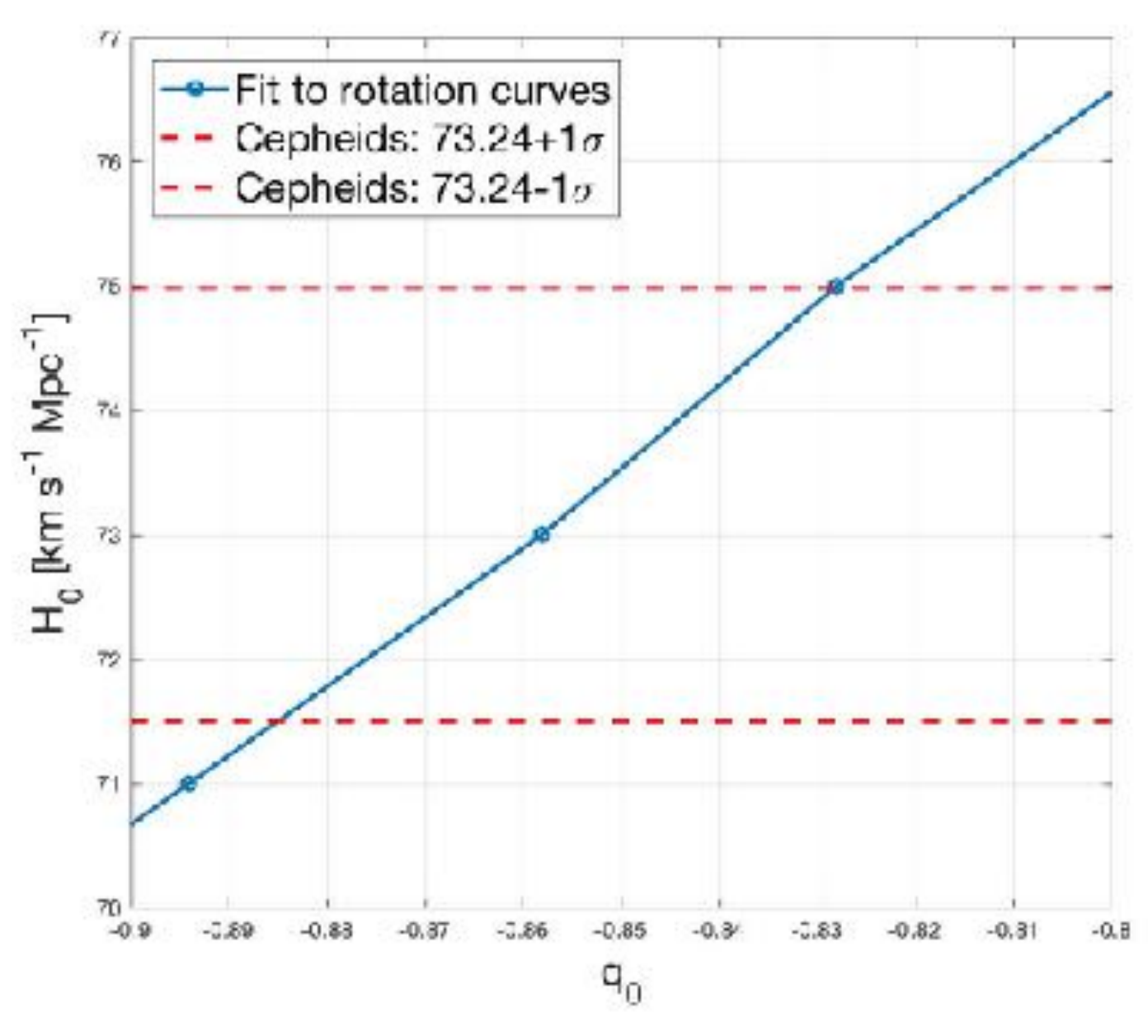

- - Riess et al. 2016 ApJ 82656 


\section{Deceleration parameter $q_{0}$}

Galaxy rotation curves

$$
q_{0}=1-\left(\frac{4 \pi a_{0}}{c H}\right)^{\frac{2}{3}} \cong-0.81
$$

integrate accelerations out to cosmological horizon

Modified FRW equations

$$
q_{0}=-\frac{1}{2}-\left.3\left(\frac{\Omega_{b}}{2 \sqrt{\pi}}\right)^{\frac{1}{2}}\right|_{\Omega_{b}=0.048} \cong-0.85
$$




\section{$Q_{0}$ from surveys of nearby spiral galaxies}

$$
Q(z)=\frac{d q(z)}{d z}=2(1-q)\left(H^{-1} \frac{d H}{d z}-a_{0}^{-1}(z) \frac{d a_{0}(z)}{d z}\right)
$$

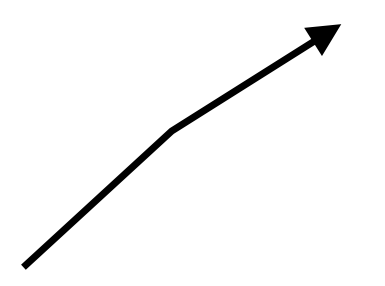

Galaxy rotation curves over, e.g., $z \approx 0-0.2$

Dynamical DE: $Q_{0}>2.5$

$\Lambda \mathrm{CDM}: Q_{0}<1$ 


\section{Conclusions}

Weak gravity governs cosmological evolution and most of galaxy dynamics. In galaxies, $1 / \mathrm{r}$ law due to perturbed inertia by collusion of Rindler and cosmological horizons (inertia $\mathrm{U}=\mathrm{mc}^{2}$ falls below $\mathrm{m}_{0} \mathrm{c}^{2}$ ).

Current data: $q_{0} \cong-0.83 \pm 0.02$

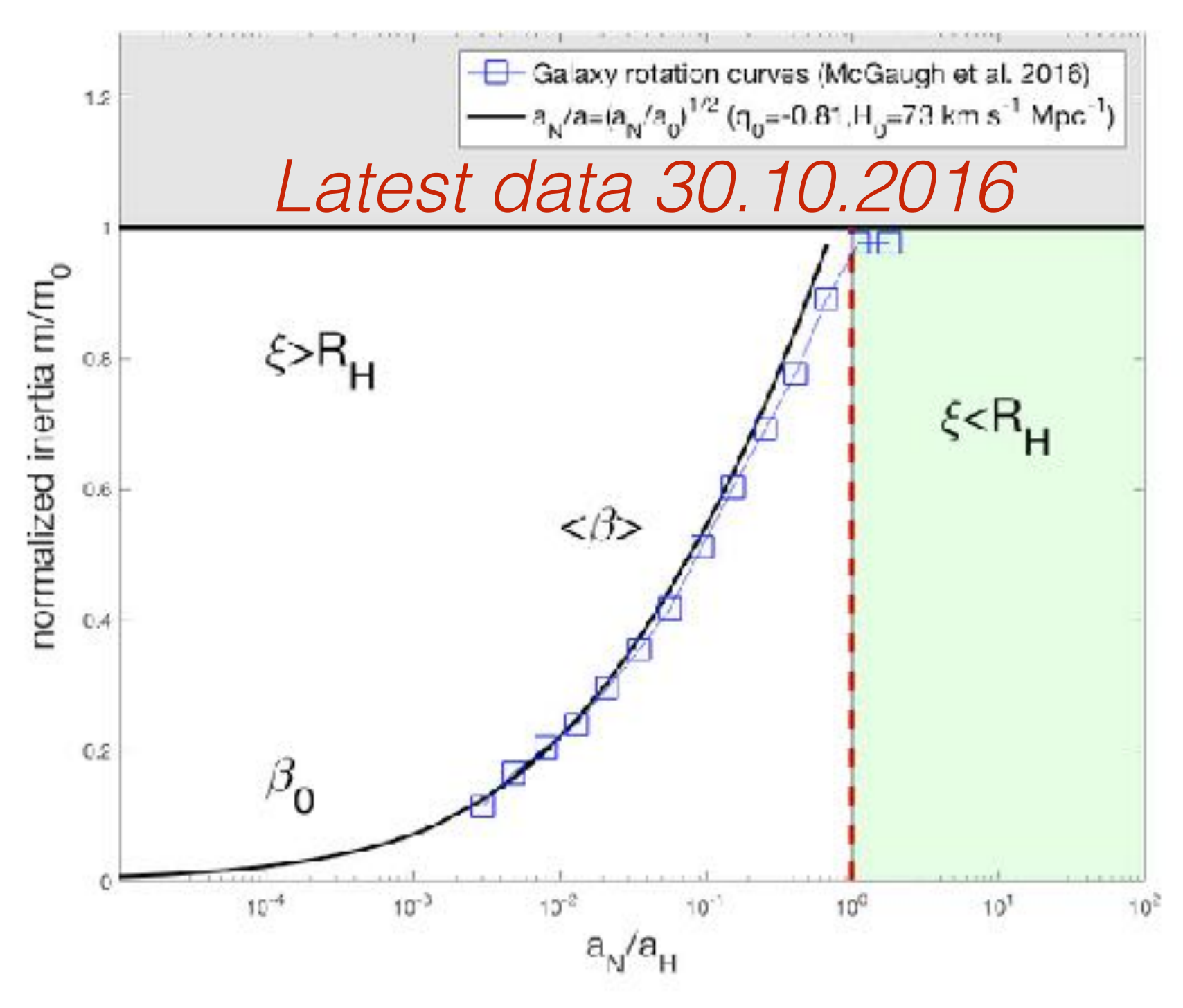

DM \& DE of holographic origin

DM is extremely light, clustering down to galaxy clusters but not much smaller. Its mass scale at the de Sitter temperature $\mathrm{k}_{\mathrm{B}} \mathrm{T} \sim 10^{-34} \mathrm{eV}$ precludes direct detection. Instead, it can be probed in free fall Cavendish type experiments in space.

$\underline{D E}$ is dynamic. Can be identified in $Q_{0}>2.5$ in surveys of nearby spiral galaxies. 